\title{
BMJ Open Tranexamic ACid during PancereaticoDuodenectomy (TAC-PD): study protocol for a multicentre randomised, blind, placebo-controlled
} trial

Kenta Ishii (D) , Yukihiro Yokoyama, Yoshihiko Yonekawa, Tomoki Ebata

To cite: Ishii K, Yokoyama Y, Yonekawa Y, et al. Tranexamic ACid during PancereaticoDuodenectomy (TAC-PD): study protocol for a multicentre randomised, blind, placebo-controlled trial. BMJ Open 2020;10:e040914. doi:10.1136/ bmjopen-2020-040914

- Prepublication history and additional materials for this paper is available online. To view these files, please visit the journal online (http://dx.doi. org/10.1136/bmjopen-2020040914).

Received 25 May 2020 Revised 05 September 2020 Accepted 14 October 2020
D) Check for updates

(c) Author(s) (or their employer(s)) 2020. Re-use permitted under CC BY-NC. No commercial re-use. See rights and permissions. Published by BMJ.

Surgical Oncology, Nagoya University Graduate School of Medicine, Nagoya, Japan

Correspondence to

Dr Kenta Ishii;

ishiikenta0701@gmail.com

\section{ABSTRACT}

Introduction Pancreaticoduodenectomy (PD) is a major gastroenterological surgery that results in a substantial amount of blood loss. Several studies have demonstrated that major blood loss during PD is associated with both short-term and long-term poor outcomes. Administration of perioperative tranexamic acid (TXA) has been reported to reduce intraoperative blood loss in various surgeries, including cardiovascular surgery and orthopaedic surgery. Nevertheless, the effect of perioperative TXA use in patients undergoing PD has not been investigated. This study aims to investigate the effect of TXA on blood loss during PD.

Methods and analysis A multicentre (six hospitals), randomised, blind (patient-blinded, surgeon-blinded, anaesthesiologist-blinded, monitor-blinded), placebocontrolled trial of TXA during PD was started in September 2019. Patients undergoing PD for biliary, duodenal or pancreatic diseases are randomly assigned to the TXA or placebo group. The stratification factors are the institutions and preoperative clinical diagnosis. Before skin incision, the participants in TXA group are administrated $1 \mathrm{~g}$ TXA as a loading infusion followed by a maintenance infusion of $125 \mathrm{mg} / \mathrm{hour}$ TXA until the end of surgery or 8hours from the incision. Participants in the placebo group are administrated the same volume of saline that is indistinguishable from the TXA. The primary outcome is blood loss during PD. The secondary outcomes are intraoperative and postoperative (up to day 2) blood transfusions, operation time, anaesthesia time, postoperative laboratory variables, length of hospital stay, in-hospital and 90-day mortality and postoperative complications occurring within 28 days of surgery or requiring readmission. To date, 115 patients of a planned 220 have been enrolled in the study.

Ethics and dissemination This protocol was approved by the Nagoya University Clinical Research Review Board and is registered with Japan Registry of Clinical Trials on 15 August 2019. The results of this trial will be disseminated through peer-reviewed journals.

Trial registration number $\mathrm{jRCTs} 041190062$.

\section{INTRODUCTION}

Pancreaticoduodenectomy (PD) is considered to be the only curative treatment option
Strengths and limitations of this study

- This randomised controlled trial (RCT) will be the first study to answer the clinical question as to whether tranexamic acid (TXA) use during pancreaticoduodenectomy (PD) is beneficial.

- A multicentre (six hospitals), blinded (patientblinded, surgeon-blinded, anaesthesiologistblinded, monitor-blinded) placebo-controlled RCT is the optimal study design for addressing the clinical question above.

- A loading dose of TXA $(1 \mathrm{~g})$ is administrated intravenously within $10 \mathrm{~min}$ before the skin incision, followed by a maintenance dose ( $125 \mathrm{mg} / \mathrm{hour})$ of continuous infusion during $\mathrm{PD}$, which are simple and easy for anaesthesiologists to administer.

- The study inclusion criteria are extensive, and exclusion criteria are limited; therefore, this protocol will have broad applicability if the trial results are positive.

- Based on the previous data regarding blood loss during PD in the participating institutions, the necessary sample size is estimated to be 220 patients; however, the amount of blood loss during PD tends to vary widely, so there remains a risk that the primary analysis will lack statistical power.

for periampullary malignancies, including pancreatic head cancer, distal bile duct cancer and duodenal cancer. Although there have been recent advancements in surgical instruments and techniques, PD is still a risky gastroenterological surgery that results in a substantial amount of blood loss and blood transfusion requirements (table 1$) \cdot{ }^{1-7}$ Recent observational studies have reported the association between both major blood loss and blood transfusions during PD and morbidity after PD. ${ }^{125}$ In addition, several studies have shown that major blood loss and blood transfusions during PD were independently 
Table 1 List of median blood loss and percentage of patients receiving perioperative blood transfusion in pancreaticoduodenectomy

\begin{tabular}{|c|c|c|c|}
\hline Authors & $\begin{array}{l}\text { Number } \\
\text { of } \\
\text { patients }\end{array}$ & $\begin{array}{l}\text { Median blood } \\
\text { loss (mL) }\end{array}$ & $\begin{array}{l}\text { Perioperative } \\
\text { transfusion } \\
(\%)\end{array}$ \\
\hline Kazanjian et al ${ }^{1}$ & 182 & 400 (ND) & 23.6 \\
\hline Braga et $a l^{2}$ & 700 & $500(300-700)$ & ND \\
\hline Ross et $a l^{3}$ & 200 & $394^{\star}$ & 18.0 \\
\hline Sutton et al ${ }^{4}$ & 697 & $672^{*}$ & 42.0 \\
\hline Seykora et $a l^{5}$ & 5323 & $400(250-600)$ & 37.2 \\
\hline Rystedt et $a l^{6}$ & 1864 & $600(300-1000)$ & $21.7 \dagger$ \\
\hline Ishii et al ${ }^{7}$ & 415 & 930 (620-1389) & $22.9 \dagger$ \\
\hline
\end{tabular}

Data of blood loss are presented as medians with interquartile ranges.

*Average blood loss is shown because the data of median blood loss are not described.

†Percentage of the patients requiring intraoperative blood transfusion is shown because the data of perioperative blood transfusion are not described.

ND, not described.

associated with poor long-term outcomes in patients with malignancies. $^{348}$

Tranexamic acid (TXA) is an antifibrinolytic agent that was first developed in 1964 and has been used in clinical practice in Japan since $1965 .{ }^{9}$ This antifibrinolytic agent is a synthetic derivative of the amino acid lysine, which reversibly binds to lysine-binding sites on plasminogen molecules, inhibits the conversion of plasminogen to plasmin and subsequently prevents the dissolution of fibrin clots. ${ }^{1011}$ Despite the lack of robust evidence of its effectiveness from clinical trials, TXA has been used routinely for many years in Japan in cases in which bleeding is a concern. However, recent large randomised clinical trials (RCTs) have demonstrated the clinical effectiveness of TXA in various settings, such as severe trauma with significant haemorrhage, ${ }^{12}$ traumatic brain injury, ${ }^{13}$ postpartum haemorrhage ${ }^{14}$ and surgery. ${ }^{15}$ Systematic reviews of clinical trials of TXA use during surgery have demonstrated that TXA significantly reduces intraoperative blood loss and the probability of receiving a blood transfusion by approximately one-third without increased risk of adverse events. ${ }^{1617}$

Although many recent clinical trials have reported the benefit of perioperative TXA use in cardiovascular, ${ }^{15} 18$ orthopaedic $^{19-21}$ and urological surgeries, ${ }^{22}$ the effect of TXA in gastroenterological surgery has been poorly investigated. Only one RCT has demonstrated the effectiveness of perioperative TXA use in patients undergoing liver surgery. ${ }^{23}$ Most importantly, to our knowledge, the effectiveness and safety of perioperative TXA use in patients undergoing PD have never been investigated.

Therefore, the objective of this study is to investigate the effectiveness and safety of TXA administration during PD using a multicentre, blind, placebo-controlled RCT. TXA was selected as the study drug because of safety profile, suitability for a blind trial and demonstrated benefits in other types of surgeries.

\section{Study objectives}

The primary objective of this RCT is to answer the clinical question as to whether intravenously administered TXA during PD is beneficial. Specifically, the effectiveness of TXA will be investigated in terms of whether it significantly reduces blood loss during PD as compared with placebo. The secondary objectives are to investigate the effect of TXA on the amount of any type of perioperative blood transfusions (up to 2 days after the operation), operation time, anaesthesia time, postoperative laboratory variables and complications occurring within 28 days of surgery or requiring readmission. The safety of TXA during PD, especially in terms of incidence of thrombotic/embolic events, will also be evaluated when these events will occur within 28 days of surgery or require readmission.

\section{METHODS AND ANALYSIS}

\section{Study design and setting}

The Tranexamic ACid during PD (TAC-PD) is a multicentre, randomised, blind, placebo-controlled trial. This study was conceived and initiated at Nagoya University Hospital, an academic referral hospital in Nagoya, Aichi, Japan. Five other referral hospitals around Nagoya (Aichi Cancer Centre, Ogaki Municipal Hospital, Toyohashi Municipal Hospital, Toyota Kosei Hospital and Kasugai Municipal Hospital) are participating in this study. Patients are randomly assigned to receive either TXA (intervention) or placebo (control) just before and during PD. Clinical practice other than the intervention will be carried out based on each institution's routine procedures. Permissive eligibility criteria, a simple study protocol and limited data collection have been adopted to maintain a pragmatic study design and study feasibility. The study design is shown in figure 1 , and the timeline of assessment and follow-up is presented in figure 2. The final report of this study will follow the Consolidated Standards of Reporting Trials statement. This study protocol was written according to the Standard Protocol Items Recommendations for Interventional Trials guidelines (see online supplemental additional file 1).

\section{Ethics, registration and informed consent}

This RCT will be conducted in accordance with the principles of the Declaration of Helsinki. In addition, because the prophylactic use of TXA in this study is considered to be an off-label use in Japan, this study is considered a specified clinical trial as defined by the Clinical Trials Act published by the Ministry of Health, Labour and Welfare of Japan. The study protocol and consent forms were reviewed as a specified clinical trial and approved by the Nagoya University Clinical Research Review Board. The ethics committee of each participating hospital also approved this study. This study was registered with 


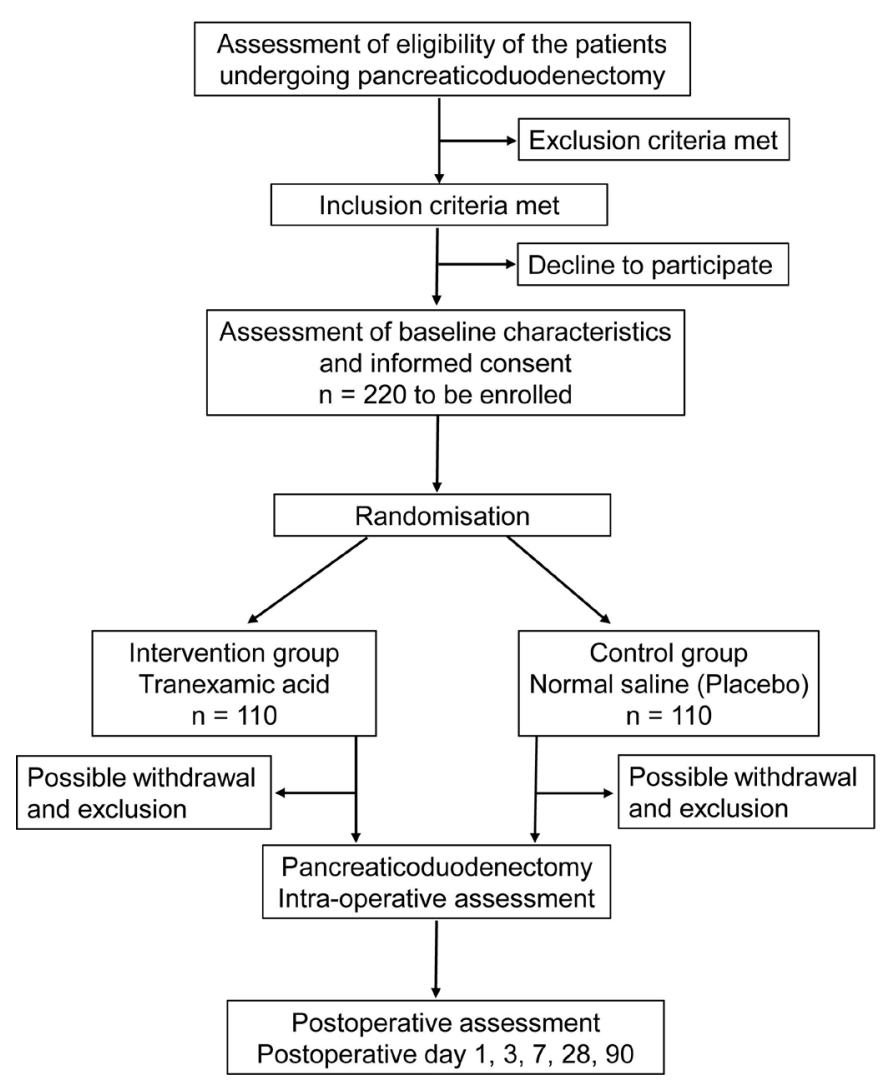

Figure 1 Study design.

the Japan Registry of Clinical Trials. Written informed consent will be obtained from all participants.

\section{Patient inclusion criteria}

Patients undergoing planned open PD for the treatment of duodenal, biliary or pancreatic disease (regardless of histology) are eligible for the study. Eligible procedures include classical PD, pylorus-preserving PD and subtotal stomach-preserving PD. PD with combined resection of the portal vein, artery and right-sided colon is also eligible. Any extent of lymph node dissection is acceptable. Eligible patients are adults (aged $\geq 20$ years) who provide written consent. Patients who have undergone previous abdominal surgery, neoadjuvant chemotherapy and/or radiation therapy are also permitted. The perioperative use of anticoagulants and antiplatelet medication is also allowed, and these agents are administered at the discretion of the surgeons and/or anaesthesiologists.

\section{Patient exclusion criteria}

Patients will be excluded from enrolment if any of the following criteria are met: (1) the planned procedure is PD with hepatectomy or other procedures for other organs (eg, colectomy for descending colon cancer, cystectomy for bladder cancer and resection of gynaecological organs), (2) the patient is receiving thrombin therapy, (3) the patient has used TXA within 7 days before surgery, (4) the patients has a known allergy to TXA, (5) the patient has a diagnosis of protein $\mathrm{C} / \mathrm{S}$ deficiency, (6) the patient has a history of symptomatic deep vein thrombosis and/ or pulmonary embolism, (7) the patient has a history of cerebral infarction and/or myocardial infarction, (8) the patient has a severe atherosclerotic lesion under treatment, (9) the patient has a history of epilepsy, (10) the patient has a chronic kidney disease (serum creatinine level $\geq 2.8 \mathrm{mg} / \mathrm{dL}$ ) or is on maintenance dialysis, (11) the patient is enrolled in another relevant interventional study. These criteria are screened by investigators just before informed consent and enrolment (within 14 days before surgery).

\section{Trial interventions}

Japanese-sourced TXA (Tranexamic acid made by Teva Takeda Pharma) is used in this study. A loading dose of TXA is administered intravenously at a dose of $1 \mathrm{~g}(10 \mathrm{~mL}$ of TXA diluted with $40 \mathrm{~mL}$ of normal saline) over $10 \mathrm{~min}$ just before surgery. TXA for maintenance dose is administered intravenously at $125 \mathrm{mg}$ /hour continuous infusion during PD (stopped at the end of surgery). If the duration of surgery is longer than 8 hours, the maintenance infusion is stopped at 8 hours from the incision (maximum dose of maintenance infusion is $1 \mathrm{~g}$ ). In the placebo group, the same amount of $0.9 \%$ sodium chloride (normal saline) is administered intravenously at the same rate. These drug (or placebo) administrations are noted on the anaesthesia record by the blinded anaesthesiologists and monitored by surgeons and other medical staff members who are also blinded to the assignment.

\section{Rationale of dose}

Previous studies on the use of perioperative TXA have used a wide variety of doses and schedules. The loading doses ranged from 10 to $40 \mathrm{mg} / \mathrm{kg}$, and maintenance doses ranged from 1 to $40 \mathrm{mg} / \mathrm{kg} /$ hour in non-cardiac surgery. ${ }^{24}$ However, there are little data regarding the perioperative use of TXA in gastroenterological surgeries. A previous open-labelled phase II trial demonstrated that the doses that will be used in our study (loading dose: $1 \mathrm{~g}$; maintenance dose: $1 \mathrm{~g} / 8$ hours) provided stable and therapeutic serum concentrations of TXA during major hepatectomy. ${ }^{25}$ Furthermore, previous large international RCTs have shown that this dose is safe. ${ }^{12}$ Despite the potential dose-dependent effect in vitro, higher doses were not adopted because of safety concerns, mainly about seizure. $^{26}$

\section{Randomisation and assignment}

Enrolled patients are randomly assigned to the TXA arm or placebo arm in a 1:1 ratio no earlier than 14 days before surgery. Investigators perform web-based online enrolment after obtaining written informed consent from the patients. Randomisation is conducted using a stochastic minimisation procedure stratified by study centres (six hospitals) and preoperative clinical diagnosis (pancreatic invasive ductal adenocarcinoma or others: ie, stratified into two groups). Investigators must provide the information of these two stratification factors with the data centre at the time of enrolment. An electronic data 


\begin{tabular}{|c|c|c|c|c|c|c|c|c|c|}
\hline & \multirow{3}{*}{\begin{tabular}{|c|} 
Enrolment/allocation \\
$\begin{array}{c}14 \text { days to } 1 \text { day } \\
\text { before surgery }\end{array}$ \\
\end{tabular}} & \multicolumn{8}{|c|}{ STUDY PERIOD } \\
\hline & & \multicolumn{5}{|c|}{ Post-allocation } & \multirow{2}{*}{\begin{tabular}{|c|} 
Close-out \\
90 days \\
postoperative
\end{tabular}} & \multicolumn{2}{|c|}{ Long-term evaluation* } \\
\hline TIMEPOINT & & Intraoperative & $\begin{array}{c}1 \text { day } \\
\text { postoperative }\end{array}$ & $\begin{array}{c}3 \text { days } \\
\text { postoperative }\end{array}$ & $\begin{array}{c}7 \text { days } \\
\text { postoperative }\end{array}$ & \begin{tabular}{|c|}
28 days \\
postoperative
\end{tabular} & & $\begin{array}{c}3 \text { years } \\
\text { postoperative }\end{array}$ & $\begin{array}{c}5 \text { years } \\
\text { postoperative }\end{array}$ \\
\hline \multicolumn{10}{|c|}{ ENROLMENT: } \\
\hline Eligibility screening & $x$ & & & & & & & & \\
\hline Informed consent & $x$ & & & & & & & & \\
\hline Allocation & $x$ & & & & & & & & \\
\hline \multicolumn{10}{|l|}{ INTERVENTIONS: } \\
\hline TXA versus Placebo & & $x$ & & & & & & & \\
\hline \multicolumn{10}{|l|}{ ASSESSMENTS: } \\
\hline $\begin{array}{r}\text { Baseline variables } \\
\text { (age, sex, height, weight, } \\
\text { disease characteristics, } \\
\text { medical history, meications, } \\
\text { preoperative laboratory variables) }\end{array}$ & $x$ & & & & & & & & \\
\hline \multicolumn{10}{|l|}{ Outcome variables } \\
\hline Intraoperative blood loss & & $x$ & & & & & & & \\
\hline Blood transfusions & & $x$ & $x$ & $x$ & & & & & \\
\hline Anaethesia and operation time & & $x$ & & & & & & & \\
\hline Postoperative laboratory variables & & & $x$ & $x$ & $x$ & & & & \\
\hline Postoperative complications & & & $x$ & $x$ & $x$ & $x$ & $x$ & & \\
\hline Adverse events & & $x$ & $x$ & $x$ & $x$ & $x$ & & & \\
\hline Length of hospital stay & & & & & & & $x$ & & \\
\hline Postoperative mortality & & & & & & & $x$ & & \\
\hline \multicolumn{10}{|l|}{ Other variables } \\
\hline Characteristics of surgery & & $x$ & & & & & & & \\
\hline Pathology & & & & & & & $x$ & & \\
\hline Recurrence-free survival & & & & & & & & $x$ & $x$ \\
\hline Overall survival & & & & & & & & $x$ & $x$ \\
\hline
\end{tabular}

Figure 2 Assessment and follow-up timeline of the TAC-PD study. TAC-PD study, Tranexamic ACid during PancreaticoDuodenectomy study; TXA, tranexamic acid. *Limited to the patients with a malignancy.

capture (EDC) system is used to perform the randomisation and data collection. The allocation sequence and the EDC were generated and managed by the data centre at Nagoya University Hospital.

\section{Blinding and drug preparation}

The results of the randomised assignment are sent to predetermined open-labelled pharmacists at each centre by email from the data centre. The open-labelled pharmacists prepare the drug or placebo at the pharmacy department on the day of the surgery. They are prohibited from providing care to the assigned patients or discussing the individual assignment with the investigators, clinical care team and patients unless serious adverse events (SAEs) such as severe allergy after the intervention occur and unblinding is requested by the anaesthesiologists and/or surgeons. Two ampoules of TXA $(2 \mathrm{~g} / 20 \mathrm{~mL})$ are mixed with $80 \mathrm{~mL}$ of normal saline, and the mixture is divided into a $50-\mathrm{mL}$ bottle for the loading dose and a 50-mL syringe for the maintenance dose when the patients are assigned to TXA group. The open-labelled pharmacists divide only $100 \mathrm{~mL}$ of normal saline into a $50-\mathrm{mL}$ bottle and a $50-\mathrm{mL}$ syringe when the patients are assigned to the placebo group. Mixing by the open-labelled pharmacists is completely out of sight of the other staff members. Anesthesiologists administer the prepared drug or placebo intravenously in the operating room. Study drugs are stored in a locked place at each centre's pharmacy department and are managed only by the open-labelled pharmacists. This protocol achieves blinding of the patients, investigators, surgeons, anaesthesiologists and other medical staff members except for the open-labelled pharmacists, because the diluted TXA is colourless and indistinguishable from the placebo. Members of the Data Safety and Monitoring Board (DSMB) are also masked the results of randomisation, and only the statistical manager at the data centre has access to the results.

\section{Other treatment}

Other techniques, drugs or equipment as well as postoperative management are administered at the discretion of the surgeons and anaesthesiologists. These include the PD approach (eg, mesenteric approach or conventional), type of reconstruction, techniques of pancreaticojejunostomy or pancreaticogastrostomy, infusion of crystalloids and colloids, blood transfusions and instrument use such as the vessel-sealing system LigaSure (Covidien Japan, Tokyo, Japan). In contrast, preoperative (within 7 days before surgery) TXA use, administration of TXA during PD outside of this trial's intervention and perioperative thrombin therapy are not allowed. 


\section{Outcome measures}

The primary outcome in this trial is blood loss during PD. Blood loss is measured and calculated based on the inout balance of the operative field. For example, amount of normal saline for intraabdominal lavage is recorded as the gain of the field, and amount of fluid in suction bottle is recorded as the loss of the field. Any fluid loss from the body such as blood-soaked gauze is weighted as precisely as possible during the operation. The secondary outcomes include operating time, anaesthesia time, whether the patient requires a blood transfusion, and the amount of the transfusion during PD and up to 2 postoperative days. To maintain the pragmatic approach in this multicentre study, strict criteria for blood transfusions were not predetermined. In general, anaesthesiologists and surgeons decide whether to transfuse based on laboratory variables and vital signs. Secondary outcomes also assess postoperative laboratory variables including white blood cell count, haemoglobin concentration, platelet count, serum creatinine, serum amylase, serum total bilirubin, C-reactive protein and variables associated with coagulation and fibrinolysis (eg, plasma fibrinogen level, prothrombin time and D-dimer). These variables are measured on postoperative days 1, 3 and 7. The morbidity, severity of complications, length of hospital stay and in-hospital as well as postoperative day 90 mortality are also evaluated as secondary outcomes. Complications include postoperative pancreatic fistula (POPF), haemorrhage associated with POPF, delayed gastric emptying (DGE), bile fistula, intra-abdominal abscess, sepsis, acute kidney injury, hepatic failure, heart failure, stromal ulcer, pneumonia, urinary tract infection and postoperative bleeding. The severity of these complications is evaluated based on the Clavien-Dindo classification system and comprehensive complication index. ${ }^{27} 28$ POPF and DGE are also classified according to the definitions of the International Study Group of Pancreatic Surgery. ${ }^{29}{ }^{30}$ Grade B or C POPF and other complications with a Clavien-Dindo score $3 \mathrm{a}$ or higher are considered clinically relevant. SAEs are defined as adverse events with a Clavien-Dindo score 4a or higher that are potentially caused by TXA, including severe drug allergy, seizure and thrombotic/embolic events such as deep venous thrombosis, pulmonary embolism, myocardial infarction and cerebral infarction. These complications and adverse events are evaluated as secondary outcomes when the events occur within 28 days of surgery or readmission is required. These outcomes (ie, blood loss, blood transfusions, operating/anaesthesia time, morbidity, SAEs, length of hospital stay and mortality) are clinically relevant for both clinicians and patients. If the patients have a pathologically proven malignancy, the long-term outcomes (recurrence-free survival and overall survival) will be assessed at 3 and 5 years after surgery.

\section{Data collection and follow-up}

Case report forms in this study are constructed as webbased forms (from the EDC). The data are entered by blinded investigators before surgery and after surgery (postoperative days 1, 3, 7, 28 and 90) and are fully supported by source documents. Baseline patient characteristics include age, sex, history, comorbidity, medications, preoperative diagnosis, preoperative laboratory variables (white blood cell count, haemoglobin concentration, platelet count, serum albumin, serum creatinine, serum amylase, serum total bilirubin, C-reactive protein, total cholesterol and variables associated with coagulation and fibrinolysis) and American Society of Anaesthesiologists physical status. The data that will be captured and relevant to the surgery include the surgical procedure, the extent of lymph node dissection, method of reconstruction and the experience of the operator. Postoperative management is performed as per-standard care at each institution; however, laboratory blood tests on postoperative days 1, 3 and 7 and drain amylase measured on postoperative day 3 are necessary to assess the secondary outcomes. Postoperative complications and their severities are judged by a blinded investigator who is independent from the patient's clinical care team. The patients will be followed up at each institution at least 90 days from the surgery. If the patients have a malignant lesion, they will be followed up or interviewed over the phone at 3 and 5 years from surgery to assess recurrence and survival.

\section{Sample size}

A previous meta-analysis has shown that TXA reduced intraoperative blood loss by $34 \%$ (95\% CI $33 \%$ to $35 \%) .{ }^{16}$ Review of past patient record at Nagoya University Hospital and other referral hospitals indicated that the average blood loss during PD was approximately $800 \mathrm{~mL}$ $(\mathrm{SD}=680 \mathrm{~mL})$. We hypothesised that the average blood loss in the TXA group will be reduced to approximately $530 \mathrm{~mL}$ ( $34 \%$ reduction from the past average). From the results of our survey and our hypothesis, assuming a power of $80 \%$ to detect an absolute difference, with a bilateral alpha risk of $5 \%$, the sample size needed is 200 patients. To account for $2 \%$ of cases being stopped before surgery (eg, due to cholangitis) and $8 \%$ incomplete PD due to tumour progression, we estimate that the final sample size required is 220 patients $(110 / \mathrm{arm})$.

\section{Statistical analysis}

Baseline characteristics of the enrolled patients will be summarised using descriptive statistics. Comparisons between the TXA group and placebo group will be performed according to an intention-to-treat principle. Student's t-test or Welch's t-test (when the data are not normally distributed) will be used to assess the primary outcome and other continuous variables included in the secondary outcomes (operating/anaesthesia time, number of transfused blood products, laboratory variables). If unacceptable baseline imbalances are present, a multiple linear regression analysis to calculate the adjusted coefficient of TXA for the blood loss will be performed. Categorical variables such as whether or not blood transfusions are required, and the occurrence of 
postoperative complications and SAEs will be evaluated using Pearson's $\chi^{2}$ test. Fisher's exact test will be applied only when the expected scores in any of the cells are $\leq 5$. Relative risk ratio and number needed to treat will also be calculated. Intention-to-treat analysis (ie, analysis population consists of all enrolled patients undergoing surgery), per-protocol analysis (ie, analysis population consists of all patients receiving complete intervention) and limited analysis (ie, analysis population consists of patients who complete the intervention and PD) will be planned. Subgroup analyses will also be planned for baseline characteristics that may affect blood loss during PD, including preoperative body mass index, diagnosis and laboratory variables. In patients with a malignancy, recurrence-free survival and overall survival will be evaluated at 3 and 5 years from surgery using Kaplan-Meier method, logrank test and Cox hazard model. Interim analysis is not planned in this trial.

\section{Unblinding}

In cases in which a severe allergy (an anaphylactic reaction), such as bronchospasm or shock, occurs during anaesthesia and TXA is suspected as the causal agent, the attending anaesthesiologists and surgeons can stop the study intervention and request emergent unblinding from the open-labelled pharmacists. If the patients received the placebo, the causal agent is not TXA but rather another drug used during anaesthesia. This unblinding protocol will allow the anaesthesiologists to properly treat the severe allergy during anaesthesia. Unblinding will also be carried out when SAEs potentially caused by TXA such as seizure and thrombotic/embolic events occur postoperatively. This unblinding is for the members of the independent DSMB and the principal investigator to properly evaluate the safety of TXA, ensure the safety of the study and report the SAEs to the Pharmaceuticals and Medical Devices Agency (PMDA) and Ministry of Health, Labour and Welfare of Japan.

\section{Individual and entire stopping rules}

A patient's participation in the trial will be stopped in the following scenarios: (1) the patient declines to participate (all enrolled patients have the right to withdraw from the trial at any time), (2) the surgery is cancelled or postponed or (3) a severe allergy during PD develops in which TXA is the suspected causal agent. The entire trial will be terminated only if it is obvious that the SAEs are occurring frequently in the TXA group. Specifically, if there are more than three patients with SAE in the TXA group than those in the placebo group, a decision of termination will be considered. This decision will be made by the members of the independent DSMB. Other early cessation rules have not been developed because interim analysis will not be planned in this trial. Data collected before the termination will be used for the analysis. In addition, SAEs that result in cessation of the trial will be analysed to evaluate the safety of TXA during PD.

\section{Trial management}

The data centre at Nagoya University Hospital is responsible for the allocation sequence, EDC design and setup, web-based online enrolment, randomisation and confidentiality of the collected data. Data of the enrolled patients are entered in the EDC and anonymised. Only the investigators, members of the data centre and site monitors have access to the EDC. The anonymised data will be retrieved from the EDC after the trial. The data and research documents will be archived for 5 years after study completion. Each institution has at least one site monitor. The site monitors have the responsibility of verifying patient eligibility, written informed consent, compliance with the protocol, accuracy of the data in the EDC and report of SAEs using on-site surveillance of source documents. The site monitors will verify the written informed consent in all participants. For other items, the first two participants will be verified, and if there is no problem, the verification will be carried out every five participants thereafter. If the site monitors find any error or deficiency, he or she presents these to the study team member, describes them in the monitoring report and implements the corrective actions. The DSMB has the responsibility of monitoring the SAEs throughout the trial. Auditing is not planned in this trial.

\section{Potential harms and reporting rule}

Data on adverse events and complications are collected in the EDC. SAEs are reported to the principal investigator by the chief investigator at each institution as soon as the SAE occurs. The principal investigator must report the occurrence of SAEs to the DSMB, PMDA and the Ministry of Health, Labour and Welfare of Japan using a specific reporting form. The DSMB will consider stopping the entire study when the incidence of the SAEs (eg, severe allergy, seizure and thrombotic/embolic events) is obviously higher in the TXA group. However, previous large studies have shown that TXA does not increase the risk of seizure or clinically relevant thrombotic/embolic events at least when the dosage of TXA is $2 \mathrm{~g}$ or less. ${ }^{12-14}$ In addition, anaphylaxis to TXA during anaesthesia is extremely rare; only a few case reports on this issue have been published. ${ }^{31-33}$ Therefore, we estimate that the probability of trial cessation due to SAEs is very low.

\section{Loss to follow-up and missing data}

In general, patients undergoing PD can be followed up until postoperative day 90 at each institution because the participating institutions are all tertiary hospitals of each region. We expect more than $99 \%$ follow-up at day 90 referring to the 90 -day follow-up completion rate $(100 \%)$ in the past observational studies. ${ }^{7}$ The available data of loss to follow-up are used for analysis, and complete case analysis will be performed if most data are present. Imputation-based approaches such as the multiple imputation method will be considered when much data are missing. Patients with a malignant lesion will generally be followed up until 3 and 5 years or death. Follow-up at 
3 and 5 years will be done by telephone interview when the patients are not in the hospital where they underwent surgery.

\section{Patients and public involvement}

Patients and/or the public were not involved in the design or conduct of this study. We will intend to actively involve patients and the public in study dissemination strategy.

\section{DISCUSSION}

The TAC-PD trial will assess whether TXA during PD will result in reduced intraoperative blood loss without increased risk of adverse events. The blood loss (not the unit of blood transfusion) was selected as the primary outcome because the decision of transfusion depends on factors such as baseline haemoglobin level other than blood loss. The effect of TXA on blood transfusion may not be an accurate indicator of the effect of TXA on surgical patients. ${ }^{16}$ The study design is a randomised, blind and placebo controlled. The stratification factors are study centres and preoperative clinical diagnosis (pancreatic invasive ductal adenocarcinoma or not). Pancreatic invasive ductal adenocarcinoma was selected as a stratification factor because some previous studies have reported that vascular resection, hard pancreas and neoadjuvant therapy, which are associated with pancreatic invasive ductal adenocarcinoma, were associated with major blood loss during PD. ${ }^{5-7}$ However, requirement for vascular resection and texture of pancreas are often uncertain before surgery, and whether performing neoadjuvant therapy largely depends on institution policy. Therefore, our pragmatic protocol includes preoperative clinical diagnosis as a stratification factor. This protocol adopted the loading dose $(1 \mathrm{~g})$ over $10 \mathrm{~min}$ just before surgery and maintenance dose $(1 \mathrm{~g})$ over 8 hours during surgery. These doses were determined based on the previous studies, ${ }^{12} 1325$ and the remaining drug at the end of surgery is not administered if the surgery finishes within 8 hours. This is because postoperative TXA use may not affect the primary outcome in this study, while there is concern that it may increase the risk of postoperative thrombotic/embolic events.

This pragmatic protocol has several potential limitations. First, although the intraoperative blood loss is measured as accurately as possible at each institution, there may be differences in blood loss measurement methods between study centres. However, study centre is a stratification factor in this trial; therefore, the difference of measurement methods is unlikely to confound the primary outcome. Second, although the sample size was estimated based on the past data of the participating institutions and the previous meta-analysis, ${ }^{16}$ there remains a risk that non-interventional factors that strongly affect the primary outcome such as vascular resection could be unbalanced because of relatively small sample size. Therefore, the data that possibly affect the blood loss are collected, and multivariate regression analysis to adjust the imbalance in two group will be planned if unacceptable baseline imbalances are present.

If the effectiveness and safety of TXA during PD are demonstrated, TXA may be almost routinely used in this clinical setting because it is both technically easy to administer and inexpensive (approximately US $\$ 1$ per patient in Japan). An economic benefit will be achieved if the use of inexpensive TXA decreases perioperative blood transfusions. To our knowledge, this trial will be the first to answer the clinical question of whether TXA use during $\mathrm{PD}$ reduces intraoperative blood loss and results in better clinical outcomes after PD. At study completion, we will better understand the benefits and risks of systemic TXA administration during PD. We expect our findings to have broad applicability, as this trial has wide inclusion criteria and narrow exclusion criteria. We hope that this trial will have a great impact not only on the establishment of TXA use during PD but also on opening the door for perioperative TXA use in gastroenterological surgeries. If the study findings are negative because of insufficient statistical power, a larger study and an RCT targeting other major gastroenterological surgeries such as major hepatectomy will be warranted.

\section{ETHICS AND DISSEMINATION}

This protocol is based on the latest version (Ver.3), as established on 13 March 2020. The study protocol was approved by the Nagoya University Clinical Research Review Board. All substantial protocol modifications will be reviewed and approved. The ethics committees of other participating hospitals (Institutional Review Board of Toyota Kosei Hospital, Clinical Research Institutional Review Board of Toyohashi Municipal Hospital, Ethics Committees of Kasugai Municipal Hospital, Clinical Research Review Committee of Ogaki Municipal Hospital and Certified Review Board of Aichi Cancer Centre) also approved this study. Investigators will obtain written informed consent from all participants. The consent forms include additional consent provisions for collection and use of participant data for future ancillary studies (see online supplemental additional file 2). The first patient was enrolled on 27 September 2019. To date (3 September 2020), 115 patients have been enrolled and successfully randomised. No SAEs have been occurred, and unblinding has never been requested. Expected completion of patient enrolment is March 2021, and data collection is to be completed in September 2021. Nagoya University Hospital undertakes randomisation, data management, statistical analysis, central trial management and coordination. The final results of this trial will be disseminated through peer-reviewed journals and conference presentations.

Acknowledgements The authors would like to thank Enago (www.enago.jp) for the English language review. We thank all surgeons, anaesthesiologist and openlabel pharmacists for their effort in conducting the TAC-PD trial. The open-label pharmacists are as follows: Yoko Ishiguro, Yosuke Niwa, Kana Ogura, Chigako Yamada, Yuko Kitahara, Rina Kishi, Noriko Takeuchi, (Nagoya University Hospital); 
Yu Kondo, Satoru Mase, Kunitoshi Toda, Azusa Hiraiwa, Kyohei Kato, Daiki Shimono (Toyota Kosei Hospital); Taeko Yaginuma, Yoichi Ando, Masahiro Okawa, Toshinori Suzuki, Tetsuhiko Harada, Masako Hashimoto, Tomohiko Kaba, Ryuichiro Suzuki, Ayami Sato, Yuki Sugita (Toyohashi Municipal Hospital); Hiroki Mizukusa, Ikko Akita, Kengo Takeuchi, Daigo Suzuki, Masatomo Ogawa, Hideaki Ishii, Yuki Ishiguro (Kasugai Municipal Hospital); Keiji Nakajima, Tetsuya Mitsuda, Shino Adachi, Kazutomo Okada, Hiroki Asano, Rika Onishi, Izumi Nagase, Tomoki Sugiyama, Erika Miyata, Hiromi Murase (Ogaki Municipal Hospital); Akimitsu Maeda, Kazuhide Inaguma, Naoya Hashimoto, Yosuke Miwa, Takuya Yamauchi, Tomoko Kato, Naoya Tomiyasu, Ikkei Shimomura, Fumiko Murata, Syuta Shibata, Naoki Sakaida, Chihaya Noritake, Toshiaki Yasukawa, Kyoko Hori (Aichi Cancer Centre).

Contributors $\mathrm{Kl}$ and $\mathrm{YY}$ conceived of and designed this trial and were responsible for drafting and submission of the manuscript. YY designed this trial and was responsible for editing the manuscript. TE had a major influence on the design of this study and helped in reviewing and revising the manuscript for intellectual content. All authors read and approved the final manuscript.

Funding The authors have not declared a specific grant for this research from any funding agency in the public, commercial or not-for-profit sectors.

Competing interests None declared.

Patient consent for publication Not required.

Provenance and peer review Not commissioned; externally peer reviewed.

Supplemental material This content has been supplied by the author(s). It has not been vetted by BMJ Publishing Group Limited (BMJ) and may not have been peer-reviewed. Any opinions or recommendations discussed are solely those of the author(s) and are not endorsed by BMJ. BMJ disclaims all liability and responsibility arising from any reliance placed on the content. Where the content includes any translated material, BMJ does not warrant the accuracy and reliability of the translations (including but not limited to local regulations, clinical guidelines, terminology, drug names and drug dosages), and is not responsible for any error and/or omissions arising from translation and adaptation or otherwise.

Open access This is an open access article distributed in accordance with the Creative Commons Attribution Non Commercial (CC BY-NC 4.0) license, which permits others to distribute, remix, adapt, build upon this work non-commercially, and license their derivative works on different terms, provided the original work is properly cited, appropriate credit is given, any changes made indicated, and the use is non-commercial. See: http://creativecommons.org/licenses/by-nc/4.0/.

ORCID iD

Kenta Ishii http://orcid.org/0000-0001-6491-5224

\section{REFERENCES}

1 Kazanjian KK, Hines OJ, Duffy JP, et al. Improved survival following pancreaticoduodenectomy to treat adenocarcinoma of the pancreas: the influence of operative blood loss. Arch Surg 2008;143:1166-71.

2 Braga M, Capretti G, Pecorelli N, et al. A prognostic score to predict major complications after pancreaticoduodenectomy. Ann Surg 2011;254:702-8.

3 Ross A, Mohammed S, Vanburen G, et al. An assessment of the necessity of transfusion during pancreatoduodenectomy. Surgery 2013;154:504-11.

4 Sutton JM, Kooby DA, Wilson GC, et al. Perioperative blood transfusion is associated with decreased survival in patients undergoing pancreaticoduodenectomy for pancreatic adenocarcinoma: a multi-institutional study. J Gastrointest Surg 2014;18:1575-87.

5 Seykora TF, Ecker BL, McMillan MT, et al. The beneficial effects of minimizing blood loss in pancreatoduodenectomy. Ann Surg 2019;270:147-57.

6 Rystedt J, Tingstedt B, Ansorge C, et al. Major intraoperative bleeding during pancreatoduodenectomy - preoperative biliary drainage is the only modifiable risk factor. HPB 2019;21:268-74.

7 Ishii K, Yokoyama Y, Ebata T, et al. Impact of the preoperative body composition indexes on intraoperative blood loss in patients undergoing pancreatoduodenectomy. Surg Today 2020

8 Maeta T, Ebata T, Hayashi E, et al. Pancreatoduodenectomy with portal vein resection for distal cholangiocarcinoma. Br J Surg 2017;104:1549-57.

9 Okamoto S, Sato S, Takada Y, et al. An active STEREO-ISOMER (TRANS-FORM) of AMCHA and its antifibrinolytic (ANTIPLASMINIC) action in vitro and in vivo. Keio $J$ Med 1964;13:177-85.
10 Nilsson IM. Clinical pharmacology of aminocaproic and tranexamic acids. J Clin Pathol Suppl 1980;14:41-7.

11 McCormack PL. Tranexamic acid: a review of its use in the treatment of hyperfibrinolysis. Drugs 2012;72:585-617.

12 Shakur H, Roberts I, Bautista R, CRASH-2 trial Collaborators. Effects of tranexamic acid on death, vascular occlusive events, and blood transfusion in trauma patients with significant haemorrhage (CRASH-2): a randomised, placebo-controlled trial. Lancet 2010;376:23-32.

13 CRASH-3 trial collaborators. Effects of tranexamic acid on death, disability, vascular occlusive events and other morbidities in patients with acute traumatic brain injury (CRASH-3): a randomised, placebocontrolled trial. Lancet 2019;394:1713-23.

14 WOMAN Trial Collaborators. Effect of early tranexamic acid administration on mortality, hysterectomy, and other morbidities in women with post-partum haemorrhage (woman): an international, randomised, double-blind, placebo-controlled trial. Lancet 2017;389:2105-16.

15 Myles PS, Smith JA, Forbes A, et al. Tranexamic acid in patients undergoing coronary-artery surgery. N Engl J Med 2017;376:136-48.

16 Ker K, Prieto-Merino D, Roberts I. Systematic review, meta-analysis and meta-regression of the effect of tranexamic acid on surgical blood loss. Br J Surg 2013;100:1271-9.

17 Ker K, Edwards P, Perel P, et al. Effect of tranexamic acid on surgical bleeding: systematic review and cumulative meta-analysis. BMJ 2012;344:e3054.

18 Dai Z, Chu H, Wang S, et al. The effect of tranexamic acid to reduce blood loss and transfusion on off-pump coronary artery bypass surgery: a systematic review and cumulative meta-analysis. J Clin Anesth 2018;44:23-31

19 Qi Y-M, Wang H-P, Li Y-J, et al. The efficacy and safety of intravenous tranexamic acid in hip fracture surgery: a systematic review and meta-analysis. J Orthop Translat 2019;19:1-11.

20 Xu S, Chen JY, Zheng Q, et al. The safest and most efficacious route of tranexamic acid administration in total joint arthroplasty: a systematic review and network meta-analysis. Thromb Res 2019;176:61-6.

21 Zhang Y, Liu H, He F, et al. Does tranexamic acid improve bleeding, transfusion, and hemoglobin level in patients undergoing multilevel spine surgery? A systematic review and meta-analysis. World Neurosurg 2019;127:289-301.

22 Crescenti A, Borghi G, Bignami E, et al. Intraoperative use of tranexamic acid to reduce transfusion rate in patients undergoing radical retropubic prostatectomy: double blind, randomised, placebo controlled trial. BMJ 2011;343:d5701.

23 Wu C-C, Ho W-M, Cheng S-B, et al. Perioperative parenteral tranexamic acid in liver tumor resection: a prospective randomized trial toward a "blood transfusion"-free hepatectomy. Ann Surg 2006;243:173-80.

24 Henry DA, Carless PA, Moxey AJ, et al. Anti-fibrinolytic use for minimising perioperative allogeneic blood transfusion. Cochrane Database Syst Rev 2011;1:CD001886.

25 Karanicolas PJ, Lin Y, Tarshis J, et al. Major liver resection, systemic fibrinolytic activity, and the impact of tranexamic acid. HPB 2016;18:991-9.

26 Lin Z, Xiaoyi Z. Tranexamic acid-associated seizures: a metaanalysis. Seizure 2016;36:70-3

27 Dindo D, Demartines N, Clavien P-A. Classification of surgical complications: a new proposal with evaluation in a cohort of 6336 patients and results of a survey. Ann Surg 2004;240:205-13.

28 Slankamenac K, Nederlof N, Pessaux P, et al. The comprehensive complication index: a novel and more sensitive endpoint for assessing outcome and reducing sample size in randomized controlled trials. Ann Surg 2014;260:757-62.

29 Bassi C, Marchegiani G, Dervenis C, et al. The 2016 update of the International Study Group (ISGPS) definition and grading of postoperative pancreatic fistula: 11 years after. Surgery 2017;161:584-91.

30 Wente MN, Bassi C, Dervenis C, et al. Delayed gastric emptying (DGE) after pancreatic surgery: a suggested definition by the International Study group of pancreatic surgery (ISGPS). Surgery 2007;142:761-8.

31 Li PH, Trigg C, Rutkowski R, et al. Anaphylaxis to tranexamic acid-A rare reaction to a common drug. J Allergy Clin Immunol Pract 2017:5:839-41.

32 Bansal RA, Nicholas A, Bansal AS. Tranexamic acid: an exceedingly rare cause of anaphylaxis during anaesthesia. Case Reports Immunol 2016;2016:1-2.

33 Chiem J, Ivanova I, Parker A, et al. Anaphylactic reaction to tranexamic acid in an adolescent undergoing posterior spinal fusion. Paediatr Anaesth 2017;27:774-5. 